\title{
Does chlorhexidine prevent dry socket?
}

\author{
Abstracted from \\ Yengopal V, Mickenautsch S. \\ Chlorhexidine for the prevention of alveolar osteitis. \\ Int J Oral Maxillofac Surg 2012; 41: 1253-1264. [Epub ahead of print] PubMedPMID: 22622144 \\ Address for correspondence: SYSTEM Initiative/Department of Community Dentistry, \\ Faculty of Health Sciences, University of the Witwatersrand, Johannesburg, South Africa.
}

\section{Question: Is chlorhexidine effective for the prevention of alveolar osteitis (AO)?}

Data sources The BBO (Bibliografia Brasileira de Odontologia), Biomed Central, Cochrane Library, Directory of Open Access Journals, LILACS, Open-J-Gate, OpenSIGLE, PubMed, Sabinet and Science-Direct databases were searched.

Study selection Articles were selected for review from the search results on the basis of their compliance with the broad inclusion criteria: relevant to the review question; and prospective two-arm (or more) clinical study. The primary outcome measure was the incidence of AO reported at the patient level.

Data extraction and synthesis Two reviewers (VY and SM) independently extracted data and assessed the quality of the accepted articles. Individual dichotomous datasets for the control and test group were extracted from each article. Where possible, missing data were calculated from information given in the text or tables. In addition, authors were contacted in order to obtain missing information. Datasets were assessed for their clinical and methodological heterogeneity following Cochrane guidelines. Meta-analysis was conducted with homogeneous datasets. Publication bias was assessed by use of a funnel plot and Egger's regression.

Results Ten randomised trials were included; almost all involved the removal of third molars. Only two of six identified application protocols (single application of chlorhexidine $0.2 \%$ gel or multiple application of $0.12 \%$ rinse versus placebo) were found to significantly decrease the incidence of $\mathrm{AO}$.

Conclusions Within the limitations of this review, only two of six identified application protocols were found to significantly decrease the incidence of $\mathrm{AO}$. The evidence for both protocols is weak and may be challenged on the grounds of high risk of selection, detection/ performance and attrition bias. This systematic review could not identify sufficient evidence supporting the use of chlorhexidine for the prevention of $\mathrm{AO}$. Chlorhexidine seems not to cause any significantly higher adverse reactions than placebo. Future high-quality randomised control trials are needed to provide conclusive evidence on this topic.

\section{Commentary}

Alveolar osteitis (AO) or dry socket is a troublesome painful complication of tooth extraction and as the authors of the review suggest, the true incidence of the condition is unknown, but estimates range from $0.5-37.5 \% .{ }^{1}$ The aim of this review was to assess the effectiveness of chlorhexidine in preventing dry socket and to identify any adverse reactions. As the authors note this is not the first review conducted of this topic, ${ }^{2-5}$ however it does have the most extensive search strategy covering ten separate databases.

Ten trials met the authors' inclusion criteria, this is two fewer than were included in the 2009 systematic review by MinguezSerra ${ }^{2}$ but that review does include non-randomised studies.

The included studies have had considerable variation in the chlorhexidine protocols used; in the format, rinse verses gel, concentrations of $0.12 \%-0.2 \%$ and the number of applications. Consequently, the authors have presented and discussed these separately. Two protocols, single application of $0.2 \%$ chlorhexidine gel and multiple applications of $0.12 \%$ chlorhexidine rinse were found to significantly decrease the incidence of $\mathrm{AO}$, but the number and quality of studies that could be included in the analysis mean that the evidence to support this is weak.

Chlorhexidine did not cause higher adverse reactions than placebo, but adverse effects were on reported in four trials.

This is a well-conducted systematic review that suffers from the variable quality of the original research, and these issues are well discussed by the authors. While a number of risk factors have been identified for AO it remains a somewhat enigmatic but wellknown complication of tooth extraction that continues to cause significant pain and distress for patients. As only three of the trials have been conducted in the last ten years there is clearly a good case for high-quality randomised controlled trials to provide clear evidence on the prevention of this painful condition.

\section{Practice points}

- There is insufficient evidence on which to recommend the use of chlorhexidine to prevent alveolar osteitis.

\section{Derek Richards}

Centre for Evidence-based Dentistry, Oxford, UK.

1. Kolokythas A, Olech E, Miloro M. Alveolar osteitis: a comprehensive review of concepts and controversies. Int J Dent 2010; 2010: 249073. Epub 2010 Jun 24. PubMed PMID: 20652078

2. Minguez-Serra MP, Salort-Llorca C, Silvestre-Donat FJ. Chlorhexidine in the prevention of dry socket: effectiveness of different dosage forms and regimens. Med Oral Patol Oral Cir Bucal 2009; 14: e445-449. PubMed PMID: 19718007.

3. Noroozi AR, Philbert RF. Modern concepts in understanding and management of the "dry socket" syndrome: comprehensive review of the literature. Oral Surg Oral Med Oral Pathol Oral Radiol Endod 2009; 107: 30-35. Epub 2008 Aug 28. Review. PubMed PMID: 18755610.

4. Hedström L, Sjögren P. Effect estimates and methodological quality of randomized controlled trials about prevention of alveolar osteitis following tooth extraction: a systematic review. Oral Surg Oral Med Oral Pathol Oral Radiol Endod 2007; 103: 8-15. Epub 2006 Apr 24. Review. PubMed PMID: 17178488.

5. Caso A, Hung LK, Beirne OR. Prevention of alveolar osteitis with chlorhexidine: a meta-analytic review. Oral Surg Oral Med Oral Pathol Oral Radiol Endod 2005; 99: 155-159. PubMed PMID: 15660084.

Evidence-Based Dentistry (2012) 13, 91. doi:10.1038/sj.ebd.6400882 